\title{
Numerical simulation of formation process of keyhole- induced pore for laser deep penetration welding
}

\author{
Pingan Shi ${ }^{\mathrm{a}}$ and Qiang Wan \\ Institute of Systemic Engineering, China Academy of Engineering Physics, Mianyang, Sichuan 621900, China
}

\begin{abstract}
In order to understand the mechanism of pore formation and accurately simulate the dynamic process of keyhole-induced pore in laser deep penetration welding, a three-phase mathematical model of laser keyhole welding is established to reflect the formation process of bubble, and a ray tracing method based on Particle level-set method is taken to track free surfaces of keyhole and pore. The results shows that the depth and shape of keyhole have an obvious characteristic of periodic changes and a phenomenon of high-frequency oscillations in the process of laser deep penetration welding, and the high-frequency oscillations of the keyhole are the main factors of laser welding instability and induced the collapse of keyhole and bubbles forming in the molten pool. These bubbles moved following the fluid flow in the molten pool, where some bubbles could escape out of molten pool under the competition of flow and solidification speed. But some bubbles captured by a solidified wall during the migration process in the molten pool would evolve into porosities. A good agreement between simulation and experimental results proved the reliability of this mathematical model, while the mechanism of pore formation can better illustrate with the model.
\end{abstract}

Keywords: laser welding; keyhole; keyhole-induced pore; formation process.

\section{Introduction}

In deep penetration laser welding, the keyhole behavior plays an important role in the formation process of pore [1]. Because of high energy density of the heat input source on the specimen, the material evaporates rapidly, and thus forms an unstable keyhole. As the liquid flow is very complex and strong oscillations can lead to fluctuates frequently of the keyhole shape, and it will influence the quality and performance of the welding joint. So it is necessary to carry out an investigation on the process and formation mechanism of pores and then provide an effective method to reduce or eliminate pore defects. However, the character of keyhole is variable during the laser welding process and it is difficult to observe a clearly dynamic process of keyhole formation based on an experiment for higher temperature and strong evaporation. Some researchers analyzed the behavior of the keyhole and the molten pool by using high-speed CCD camera, spectral analysis, and sound monitoring [2-4]. These observations provided helpful information for understanding the dynamic process of keyhole, but some details of this process need to be revealed further. And the underlying physics such as changes of temperature caused by heating and cooling and flow field during the laser process could not be revealed just based on experimental research, which would determine the pore formation. The

a Corresponding author : shipa@caep.cn

(C) 2016. The authors - Published by Atlantis Press 
multiple physical problems for the forming process of pore in deep penetration laser welding could be well illustrated by means of a numerical simulation based on a comprehensive mathematical model. However, the simulation of porosities in deep penetration laser welding is rarely involved. Zhou and Tsai [5] built a mathematical model considering both the welding pool and plasma zone, to simulate the pore formation process during pulsed laser welding process. It was pointed out that pore would be formed when the solidification rate of a molten metal exceeded the backfilling speed of the molten metal, but the model was only for static welding process. Li et al [6] simulated the dynamic process of keyhole and pore formation by means of volume-of-fluid (VOF) method and homogenous bubble model. The results showed that the unstable keyhole formed and the complex fluid flow inside the molten pool led to the collapse of the keyhole and then induced bubbles to occur in the molten pool. Nevertheless, the influence of plasma on keyhole behavior was neglected and the formation process and mechanism of pore were not explicitly investigated. Pang et al [7] had simulated and the processes of three-dimensional periodical keyhole oscillation and bubble formation in the laser welding process by using a three-dimensional sharp interface model. By calculation, the mechanism of pore formation was studied, but the pore formation process mechanism is still not well clarified until now. In this paper, a three-dimensional self-consistent model was proposed to simulate the dynamic process of pore formation. The effects of varied physical and thermodynamic factors were all considered in this integral model. Transient processes of bubble formation were simulated and discussed. The research results of this paper could better clarify the pore formation process in deep penetration laser welding and also help to put forward a method to eliminate porosities.

\section{Model description}

The governing equations for mass, momentum and energy conservation are expressed as follows:

$$
\begin{gathered}
\frac{\partial \rho}{\partial t}+\nabla \cdot(\rho \mathbf{V})=0 \\
\rho\left(\frac{\partial \mathbf{V}}{\partial t}+(\mathbf{V} \cdot \nabla) \mathbf{V}\right)=\nabla \cdot(\mu \nabla \mathbf{V})-\nabla \mathbf{p}-\frac{\mu}{K} \mathbf{V}-\frac{C_{p} \rho}{\sqrt{K}}|\mathbf{V}| \mathbf{V}+\rho \mathbf{g} \beta\left(T-T_{0}\right) \\
\rho C_{p}\left(\frac{\partial T}{\partial t}+(\mathbf{V} \cdot \nabla) T\right)=\nabla \cdot(\lambda \nabla T)
\end{gathered}
$$

where $\mathbf{V}=(u, v, w)$ is the velocity vector, $\mu$ is the viscosity of fluid flow, $\rho$ is the density, $\mathbf{p}$ is the pressure, $\mathbf{g}$ is the gravitational vector, $\beta$ is the thermal expansion coefficient, $C_{p}$ and $\lambda$ is the thermal capacity and thermal conductivity, $T_{0}$ the reference temperature. $K$ is the Carman-Kozeny coefficient, which is related to the liquid volume fraction $f_{l}(T)$ and can be described as follows:

$$
K=\frac{f_{l}^{3}(T) d^{2}}{180\left(1-f(T)_{l}\right)^{2}}
$$

where $d$ is proportional to the dendrite dimension, which is assumed to be a constant and in the order of $10^{-2} \mathrm{~cm}$. The liquid volume fraction of $f_{l}(T)$ is deduced from temperature and is assumed to vary linearly between the solidus and liquidus temperatures [7]. The liquid volume fraction of $f_{l}(T)$ is deduced from temperature and is assumed to vary linearly between the solidus and liquidus temperatures. The liquid volume fraction $f_{l}(T)$ can be defined as: 


$$
f_{l}(T)= \begin{cases}1 & T>T_{\mathrm{c}}+\Delta T \\ \frac{T-T_{s}}{T_{l}-T_{s}} & T_{\mathrm{c}}-\Delta T \leq T \leq T_{\mathrm{c}}+\Delta T \\ 0 & T<T_{\mathrm{c}}-\Delta T\end{cases}
$$

where, $T_{\mathrm{c}}=\left(T_{\mathrm{s}}+T_{1}\right) / 2, T_{\mathrm{s}}$ and $T_{1}$ represent solidus and liquidus temperature. $T_{\mathrm{c}}-\Delta T$ and $T_{\mathrm{c}}+\Delta T$ are the phase change points.

The level-set method is used to track the keyhole free surface. With this technique, the keyholefree surface at an arbitrary welding time is modeled as the zero contour of an implicit function: $\phi(\mathbf{x}, t):\left(R^{3}, t\right) \rightarrow R$ defined in the entire physical domain. The implicit function is generally defined as the closet signed distance $d(\mathbf{x}, \Omega)$ from the cell center to the moving interface $\Omega$. The keyhole-free surface at an arbitrary welding time can be described as follows:

$$
\phi(\mathbf{x})= \begin{cases}-d(\mathbf{x}, \Omega) & x \in \Omega_{\mathrm{n}} \\ 0 & x \in \Omega \\ d(\mathbf{x}, \Omega) & x \in\left(R^{3}-\Omega-\Omega_{\mathrm{n}}\right)\end{cases}
$$

where $\Omega_{\text {in }}$ and $\left(R^{3}-\Omega \Omega\right.$ in $)$ denote the metal liquid domain and gas fluid domain, respectively. The keyhole interface position can be described as:

$$
\frac{\partial \phi}{\partial t}+\mathbf{V} \cdot \nabla \phi=0
$$

where $\mathbf{V}$ is the local fluid flow velocity inside the molten pool. The level set value may fail to be a distance function after being advanced by Eq.(4). Therefore, this value must be reinitialized every time step to keep it as a signed distance field. The re-initialization process can be formulated as below static Hamilton-Jacobi problem:

$$
|\nabla \phi|=1
$$

The level-set technique simplifies the calculations of the geometrical properties, such as curvature and normal, of the keyhole free surface. With the level-set technique, normal $\mathbf{n}$ and curvature $\kappa$ of free surface can be described:

$$
\mathbf{n}=\nabla \phi /|\nabla \phi|, k=\nabla \cdot \mathbf{n}
$$

After the keyhole-free surface is determined, a robust ray tracing method is used to calculate the laser density due to multiple reflection absorptions [7]. By the ray tracing method, for a given arbitrary point on the keyhole-free surface, the absorbed energy density $q_{\text {beam }}$ can be formulated as:

$$
\begin{gathered}
q_{\text {beam }}=I_{0}(r, z)\left(\mathbf{I}_{0} \cdot \mathbf{n}_{0}\right) \alpha_{F r}\left(\theta_{0}\right)+\sum_{m=1}^{N} I_{m}(r, z)\left(\mathbf{I}_{m} \cdot \mathbf{n}_{m}\right) \alpha_{F r}\left(\theta_{m}\right) \\
\alpha_{F r}(\theta)=1-\frac{1}{2}\left[\frac{1+(1-\varepsilon \cos \theta)^{2}}{1+(1+\varepsilon \cos \theta)^{2}}+\frac{\varepsilon^{2}-2 \varepsilon \cos \theta+2 \cos ^{2} \theta}{\varepsilon^{2}+2 \varepsilon \cos \theta+2 \cos ^{2} \theta}\right]
\end{gathered}
$$


where $\theta$ is the angle between the incident beam and the normal of the point on the keyhole wall, $\alpha_{F r}$ is the Fresnel absorption coefficient, $N$ the incident times of beams after multiple reflections, I the unit vector of incident beams, $\mathbf{n}$ the unit normal vector, $\varepsilon$ a coefficient related to the types of lasers, $I_{0}(r, z)$ the beam energy density distribution before irradiation and $I_{m}(r, z)$ the beam energy density of the $m$ th reflections. In this study, the laser density is assumed to be Gaussian and does not attenuate along the penetration direction perpendicular to the specimen. The distribution equation of laser beam energy density can be described as

$$
I_{0}(r, z)=3 Q /\left(\pi R^{2}\right) \exp \left(-3 r^{2} / R^{2}\right)
$$

where $R$ is the beam radius and $Q$ the power density.

\section{Physical properties}

Since the transient keyhole evolution, heat transfer and fluid flow behavior in laser welding are solved by coupled methods, so the time step will be very small in the simulation. In order to make the calculation tractable, the small physical size of the specimens, $4 \mathrm{~mm} \times 2 \mathrm{~mm} \times 2 \mathrm{~mm}$ in the welding, cross section and penetration directions, respectively, is thus employed in the simulation. In the simulation, some extra computational grids of vacuum on the top specimen as well as below the specimen in the penetration direction are used to track the free surface deformations. A uniform cell scheme is used here, and the cell size is $1 / 10$ of the radius of the laser beam. The physical properties and machining parameters are referred to references [8]. A laser power of $1500 \mathrm{~W}$ and a beam diameter of $0.2 \mathrm{~mm}$ were taken in the laser welding process. The laser was assumed to travel along the $\mathrm{x}$-direction at the velocity of $3 \mathrm{~m} / \mathrm{min}$. The calculation time is about $0.6 \mathrm{~s}$, which is enough for solidification after the melting process and evaporation.

\section{The formation process of the bubbles}

A sequence of pictures in Fig. 1 shows the formation process of a bubble. At $t=17.453 \mathrm{~ms}$, it can be seen that the molten metals flowed violently in the rear part of the molten pool, and humps caused by the back-flow melt occur at the back wall of the keyhole. Then, the keyhole begins to oscillate severely as the humps collapse as shown in Fig. 1 at $t=17.567 \mathrm{~ms}$, causing closed spaces inside the keyhole separated by liquid bridges. But the molten metal in the middle of the keyhole flowed downwards rapidly under the effect of the recoil pressure, which would lead to the opening of the keyhole again. At $t=17.786 \mathrm{~ms}$, the bottom part of the keyhole form an obvious depression in the rear wall of the keyhole, and a downward flow of the molten metal above the depression could provide the formation condition of bubble. A bubble formed near to the bottom of the molten pool as shown in Fig. $1 \mathrm{~d}$. As the collapse of the keyhole periodically occurred, there will be a lot of the bubbles periodically forming at the lower part of the keyhole. It can be seen that the formation process of the bubbles was closely related to the flow patterns in the rear part of the molten pool.

In general, the generating of the bubbles results from three ways: the first way is the bubble escaping from the surface of the molten pool, the second way is the bubble captured by the solidification interface during the movement process, becoming a pore, and the third way is the bubble formed in the mushy zone remains as a pore. So maintaining the keyhole stable and making the bubbles escape from the molten pool timely are very helpful to eliminate pore. 


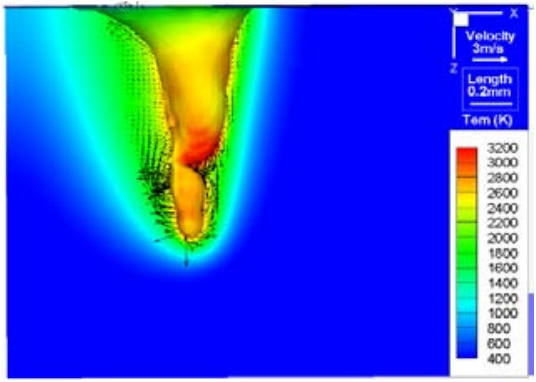

(a) $17.453 \mathrm{~ms}$

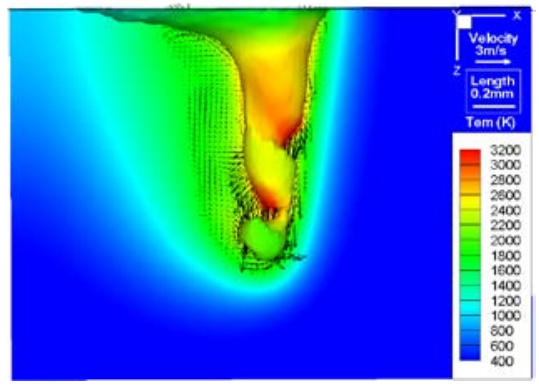

(c) $17.786 \mathrm{~ms}$

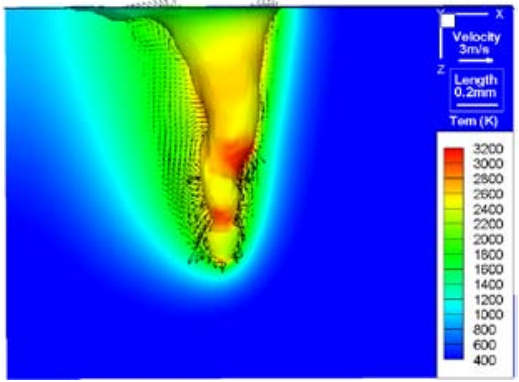

(b) $17.567 \mathrm{~ms}$

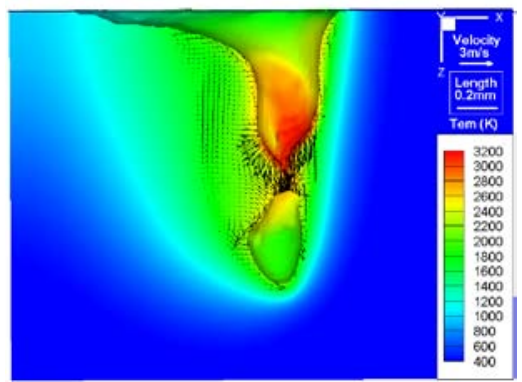

(d) $18.006 \mathrm{~ms}$

Figure 1. Transient Behavior of bubble formation during deep penetration laser welding

\section{Summary}

In order to investigate the formation process of the keyhole-induced pore and understand the mechanism of pore formation in deep penetration laser welding. A three-phase unification mathematical model of laser keyhole welding is established to combine the effects of threedimensional transient keyhole and transient molten pool. Meanwhile, the calculation results based on this model is verified through the experiment results. The major findings of the present theoretical modeling study are listed as follows:

(1)The physics of three-dimensional periodical keyhole instability and bubble formation in deep penetration laser welding can be predicted, and thus the mechanism of pore formation was better clarified with this model.

(2)The bubbles formed at different locations presented quite different migration behaviors in the molten pool. The migration of bubbles is mainly affected by the surrounding fluid flow and the buoyancy. Some bubbles might merge with other bubbles during the process of migration.

(3)Some bubbles could escape out of the molten pool under the competition of flow and solidification speed, but some bubbles captured by the solidified wall during the migration process in molten pool would evolve into porosities. It is also found that some bubbles forming adjacent to the fusion line are easier to be captured by the solidification surface, which could explain some porosities occurring close to the fusion line.

\section{Acknowledgement}

This work is supported by the Key Foundation of China Academy of Engineering Physics (No. 2013A0203008) and the key subject "Computational Solid Mechanics" of China Academy of Engineering Physics. Work partially supported by grant 11372295 of the National Natural Science Foundation of China. 


\section{References}

1. Aalderinka B J, Aarts R, de Lange D F, et al, Experimental observation of keyhole shapes in the laser welding of aluminum blanks, J Laser Appl. 19, 4 (2007), pp. 245-251.

2. Farson D F, Ali A, Li X C, Laser weld penetration monitoring with multiple emission signal measurements, J Laser Appl. 11, 2(1999), pp. 47-53.

3. Sanders P G, Leong K H, Keske J S, et al, Real-time monitoring of laser beam welding using infrared weld emissions, J Laser Appl. 10, 5(1998), pp. 205-211.

4. Farson D F, Kim K R, Generation of optical and acoustic emissions in laser weld plumes, J Appl Phys. 85, 3(1998), pp. 1329-1336.

5. Zhou J, Tsai H L and Lehnhoff T F, Investigation of transport phenomena and defect formation in pulsed laser keyhole welding of zinc-coated steels, J. Phys. D: Appl. Phys. 39, 24(2006), pp. 5338-5355.

6. Li X B, Lu F G, Cui H C, Numerical modeling on the formation process of keyhole-induced pore for laser welding steel with T-joint, Int J Adv Manuf Technol, 72 (2014), pp. 241-254.

7. Pang S, Chen L, Zhou J, A three-dimensional sharp interface model for self-consistent keyhole and molten pool dynamics in deep penetration laser welding, J. Phys. D: Appl. Phys. 44, 2(2011), pp. 025301/1-025301/15.

8. Zhao H, Niu W, Zhang B, Modelling of keyhole dynamics and pore formation considering the adaptive keyhole shape and three-phase coupling during deep penetration laser welding, $J$ Phys $D$ Appl Phys. 44, 48(2011), pp. 1-15. 